\title{
A Case of Nasu-Hakola Disease without Fractures or Consanguinity Diagnosed Using Exome Sequencing and Treated with Sodium Valproate
}

\author{
Kiyohiro Yamazaki, Yuta Yoshino, Yoko Mori, Shinichiro Ochi, Taku Yoshida, Takashi Ishimaru, Shu-ichi Ueno \\ Department of Neuropsychiatry, Ehime University Graduate School of Medicine, Toon, Japan
}

\begin{abstract}
Nasu-Hakola disease (NHD) is a rare autosomal recessive neuropsychiatric disorder characterized by bone cysts, fractures, and cognitive impairment. Two genes are responsible for the development of NHD; TYROBP and TREM2. Although it presents with typical signs and symptoms, diagnosing this disease remains difficult. This case report describes a male with NHD with no family or past history of bone fractures who was diagnosed using exome sequencing. His frontal lobe psychiatric symptoms recovered partially following treatment with sodium valproate, but not with an antipsychotic.
\end{abstract}

KEY WORDS: Nasu-Hakola disease; Exome sequencing; TYROBP (DAP12), TREM2, Frontal lobe symptoms; Sodium valproate.

\section{INTRODUCTION}

Polycystic lipomembranous osteodysplasia with sclerosing leukoencephalopathy, better known as Nasu-Hakola disease (NHD), is a rare autosomal recessive disease. ${ }^{1)}$ The TYROBP and TREM2 genes are responsible for $\mathrm{NHD},{ }^{2,3)}$ which is characterized by multiple bone cysts, bone fractures, and frontal lobe symptoms, such as disinhibition, euphoria, progressive dementia, myoclonic twitches, convulsions, and gait disturbances. ${ }^{1,2,4)}$ This case report describes a man diagnosed with NHD using exome sequencing analysis to identify a homozygous mutation of TYROBP (DAP12) c.141 del G in exon 3. Second-generation exome sequencing is an inexpensive, reliable method for detecting inherited disorders ${ }^{4)}$ and is more convenient than whole genome sequencing. ${ }^{5,6)}$ Since there is no pharmacological cure for NHD, sodium valproate was administered to treat his frontal lobe symptoms, which included abnormal behaviors. This treatment regimen was more effective than the use of atypical antipsychotics alone.

\footnotetext{
Received: March 9, 2015/Revised: April 21, 2015

Accepted: May 18, 2015

Address for correspondence: Kiyohiro Yamazaki

Department of Neuropsychiatry, Ehime University Graduate School of Medicine, Shitsukawa, Toon, Ehime 791-0295, Japan

Tel: +81-89-960-5315, Fax: +81-89-960-5317

E-mail: khryamazaki5555@gmail.com
}

CASE

A 39-year-old Japanese man with frontal lobe symptoms, including stereotyped and socially inappropriate behaviors, disinhibition, and euphoria, was admitted to our university hospital in November 2011. Over the previous 2 years, he had shown slight impairment in recent memory and abnormal behaviors, such as reading comics during a parent-teacher association meeting and pilfering. In the hospital, the patient was secured in a unit within the psychiatric ward because he entered other rooms without permission. Subsequently, he repeatedly touched female nurses in an inappropriate manner.

The patient's Mini-Mental State Examination score was 23/30 and his Frontal Assessment Battery score was $12 / 18$. His deep tendon reflexes in the extremities were brisk and the Babinski sign was positive bilaterally, but he did not have any extrapyramidal symptoms. A blood examination revealed no abnormalities other than subtle liver dysfunction, with an aspartate transaminase level of 77 IU/L (normal range 9-37 IU/L), alanine transaminase of $176 \mathrm{IU} / \mathrm{L}$ (normal range 3-49 IU/L), iron deficiency (106 $\mu \mathrm{g} / \mathrm{dl}$; normal range 120-140 $\mu \mathrm{g} / \mathrm{dl})$ and an elevated ferritin $(656 \mathrm{ng} / \mathrm{ml}$; normal range $27-211 \mathrm{ng} / \mathrm{ml})$. Brain fluid-attenuated inversion recovery magnetic resonance imaging revealed diffuse high-intensity white matter areas and slightly diffuse atrophy in the cerebral cortices without the "eye of the tiger" sign (Fig. 1). No abnormalities were detected in the cerebrospinal fluid or on

(a) This is an Open-Access article distributed under the terms of the Creative Commons Attribution Non-Commercial License (http://creativecommons.org/licenses/by-nc/4.0) which permits unrestricted non-commercial use, distribution, and reproduction in any medium, provided the original work is properly cited. 

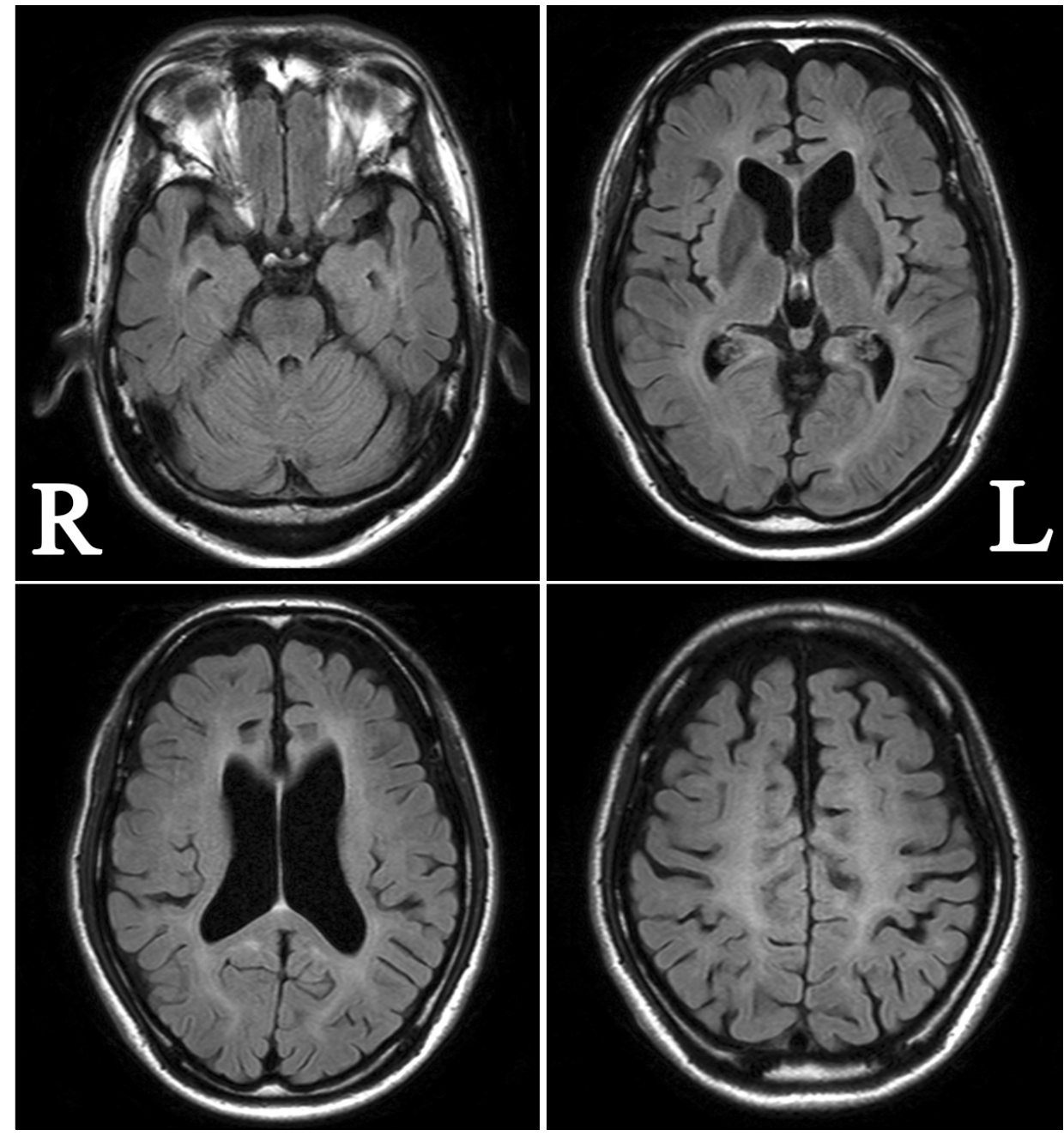

Fig. 1. Brain fluid-attenuated inversion recovery magnetic resonance imaging shows high intensity areas in periventricular white matter, and fronto-temporal lobe atrophy.

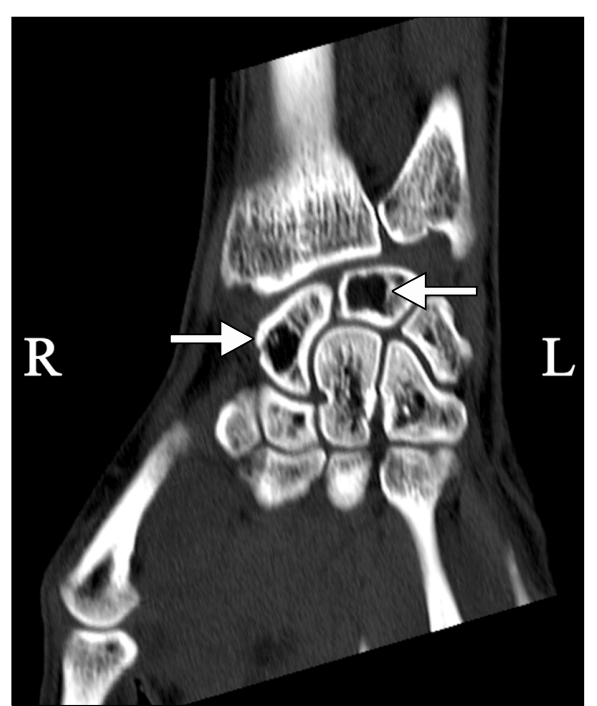

Fig. 2. Bone cysts in carpals (e.g., navicular, lunate bone) of the left wrist. electroencephalography.

Although he had no family history of NHD, a genetic analysis was performed for several inherited neurological disorders, including autosomal dominant familial Alzheimer's disease (amyloid precursor protein, presenilin 1, microtubule-associated protein tau, and progranulin) genes known to cause neurodegeneration in the brain due to iron accumulation (PANK2, C19orf12, and $F T L$ ), and leukoencephalopathy (CSF1R); all were found to be negative. Subsequently, exome sequencing conducted in the patient and his parents identified a mutation in the TYROBP (DAP12) gene, located on chromosome 19q13.1. The mutation was a homozygous single-base deletion of $141 \mathrm{G}$ in exon 3 that was observed in the patient's genomic DNA. His parents were each heterozygous for the mutation. As a result, the patient was diagnosed with NHD. After making a definitive diagnosis, a retrospective orthopedic analysis of computed tomography of the patient's hand (obtained when his left wrist was twisted be- 
fore the neuropsychiatric symptoms appeared) showed bone cysts. To date, he has not suffered any bone fractures (Fig. 2).

To address his frontal lobe symptoms, the patient was first given risperidone, increasing the dose slowly from 1 to $3 \mathrm{mg}$ /day over 4 months. However, the risperidone dose was reduced because it had little effect, but caused drug-induced parkinsonian behaviors, such as sialorrhea and postural instability. Subsequently, he was given 400 $\mathrm{mg}$ of sodium valproate (serum valproic acid concentration $49.46 \mu \mathrm{g} / \mathrm{ml}$ ) together with risperidone $1 \mathrm{mg} /$ day. Beginning approximately 2 weeks after starting the sodium valproate therapy, the patient's socially inappropriate behaviors improved gradually, he was allowed to roam the psychiatric unit freely, and there were no obvious side effects. Presently, the patient has been hospitalized for 3 years and his cognitive impairment has progressed rapidly. We can no longer communicate with him.

\section{DISCUSSION}

Since NHD is such a rare, progressive neurodegenerative disease, no effective treatment regimen has been established. The activity of patient described here is markedly limited due to the nature of his frontal lobe symptoms. Risperidone ( $3 \mathrm{mg} /$ day) effectively treated these symptoms, but caused several intolerable adverse effects; subsequently, risperidone $1 \mathrm{mg}$ /day was combined with sodium valproate $400 \mathrm{mg} /$ day.

Sodium valproate may be useful for treating behavioral disturbances in patients with dementia, ${ }^{7)}$ although a recent meta-analysis indicated that sodium valproate cannot be recommended as a first-line treatment for demented patients with agitation. ${ }^{8)}$ However, the subjects in this meta-analysis were 65 years of age and older and were combined in a common dementia category regardless of type. We suggest that NHD patients not be considered demented, as in previous case reports. In addition, our patient had no convulsions, possibly due to the sodium valproate treatment, although convulsions may present at an earlier age or be more common in other patients with TYROBP mutations." Alternatively, cognitive enhancers such as donepezil, galantamine, rivastigmine, or memantine could have been used to treat this patient because these drugs promote neurogenesis in the central nervous system, ${ }^{10,11)}$ which might prevent the progression of NHD.

It is unusual for NHD to occur without bone fractures or bone pain and homozygous mutations are rarely reported in NHD patients, although our patient's parents were not consanguineous. Our patient was diagnosed with NHD using exome sequencing, which is a powerful diagnostic tool when a patient has an inherited disease without a family history. Regardless, a patient's complete medical information should be obtained before performing exome sequencing in order to improve the accuracy of the clinical diagnosis. In this case, NHD might have been diagnosed earlier if the patient's existing orthopedic medical records had been examined.

\section{Acknowledgments}

We thank the Japan Community Healthcare Organization Uwajima Hospital, and Kuroda Hospital for providing his information in detail.

\section{REFERENCES}

1. Hakola HP. Neuropsychiatric and genetic aspects of a new hereditary disease characterized by progressive dementia and lipomembranous polycystic osteodysplasia. Acta Psychiatr Scand Suppl 1972;232:1-173.

2. Paloneva J, Kestilä M, Wu J, Salminen A, Böhling T, Ruotsalainen $\mathrm{V}$, et al. Loss-of-function mutations in TYROBP (DAP12) result in a presenile dementia with bone cysts. Nat Genet 2000;25:357-361.

3. Paloneva J, Manninen T, Christman G, Hovanes K, Mandelin J, Adolfsson R, et al. Mutations in two genes encoding different subunits of a receptor signaling complex result in an identical disease phenotype. Am J Hum Genet 2002;71:656-662.

4. Madry H, Prudlo J, Grgic A, Freyschmidt J. Nasu-Hakola disease (PLOSL): report of five cases and review of the literature. Clin Orthop Relat Res 2007;454:262-269.

5. Biesecker LG, Green RC. Diagnostic clinical genome and exome sequencing. N Engl J Med 2014;370:2418-2425.

6. Teer JK, Mullikin JC. Exome sequencing: the sweet spot before whole genomes. Hum Mol Genet 2010;19:R145R151.

7. Mellow AM, Solano-Lopez C, Davis S. Sodium valproate in the treatment of behavioral disturbance in dementia. $J$ Geriatr Psychiatry Neurol 1993;6:205-209.

8. Lonergan E, Luxenberg J. Valproate preparations for agitation in dementia. Cochrane Database Syst Rev 2009; (3): $C D 003945$.

9. Klünemann HH, Ridha BH, Magy L, Wherrett JR, Hemelsoet DM, Keen RW, et al. The genetic causes of basal ganglia calcification, dementia, and bone cysts: DAP12 and TREM2. Neurology 2005;64:1502-1507.

10. Kotani S, Yamauchi T, Teramoto T, Ogura H. Donepezil, an acetylcholinesterase inhibitor, enhances adult hippocampal neurogenesis. Chem Biol Interact 2008;175:227-230.

11. Ishikawa R, Kim R, Namba T, Kohsaka S, Uchino S, Kida $\mathrm{S}$. Time-dependent enhancement of hippocampus-dependent memory after treatment with memantine: Implications for enhanced hippocampal adult neurogenesis. Hippocampus 2014;24:784-793. 